\title{
Interacting with Casework Documents Using Timelines
}

\author{
Morten Bohøj and Niels Olof Bouvin \\ Department of Computer Science, Aarhus University \\ Åbogade 34, 8200 Århus N, Denmark \\ bohoej@cs.au.dk, bouvin@Cs.au.dk
}

\begin{abstract}
We present a way of creating an overview of administrative procedures using timelines. Our design also provides for manipulation of the information given on the timeline through interacting directly with the timeline. As an example application, a prototype supporting administrative procedures surrounding parental leave in Denmark, has been developed and evaluated.
\end{abstract}

Keywords: Timeline, visualization, manipulation, casework.

\section{Introduction}

We present a method of using timelines to visualize administrative procedures in the public sector and a way of interacting with the timeline. The visualization of administrative procedures may be interesting in itself, but the main contribution of his work is the interaction with and manipulation of the timeline. The application is a web based application constructed using the Google Web Toolkit.

As a demonstrator for our approach, we have chosen to focus on an application for parental leave, as this is a complex administrative procedure under Danish law. In Denmark you are entitled to leave subsidized by the municipality if you are working. This leave consists of a variety of leave types e.g. four weeks of maternal pregnancy leave and two weeks of paternal postnatal leave. A total of 52 subsidized weeks to be split between the parents are available. These 52 weeks can be extended with 8 or 14 weeks if taken as direct extension to the 52 weeks, but with the same amount of money spread over a greater time span. The 52 weeks can be spread over the first nine years of the child's life, allowing for options such as two hours every other Friday. The systems available today at the municipality cannot handle this flexibility very well and case workers try to adapt by counting days in paper calendars and starting/stopping cases manually. There is no system for the parents to keep track of their leave, only if they themselves keep track.

Our design gives easier access for parents to information and this way takes some of the workload from the caseworkers. Our approach is not limited to handling parental leave applications and can be generalized to other casework applications.

The work presented herein was done under the auspices of the eGov+, a project looking into ways of using web technologies to improve communication between the citizens and the Danish public sector. 


\section{Related Work}

Timelines are well suited to represent chronologically occurring events, and several systems and UI widget sets have in recent years been developed to exploit this on the web. The focus of most systems are to facilitate browsing with some support for authoring timelines for others to read. A typical example of this class of timeline sites is TimeRime [1], which supports authoring of rich media timelines (rich text, images, and links) and sharing of the same. Other systems emphasis the sharing of timelines, as seen on TimelineIndex [2], which support embedding timelines on other websites. The technologies vary - many systems have previously used Flash (as seen in e.g., TimeGlider [3]), yet modern UI widget sets such as SIMILE Timeline [4] demonstrates what can be accomplished in a modern web browser.

While these systems excel in visualizing chronological events, they are not intended as tools for other activities rather than the presentation of the timeline itself. This is one of the defining differences to the work reported herein, as we actively explore the timeline as the locus of collaborative activity and planning.

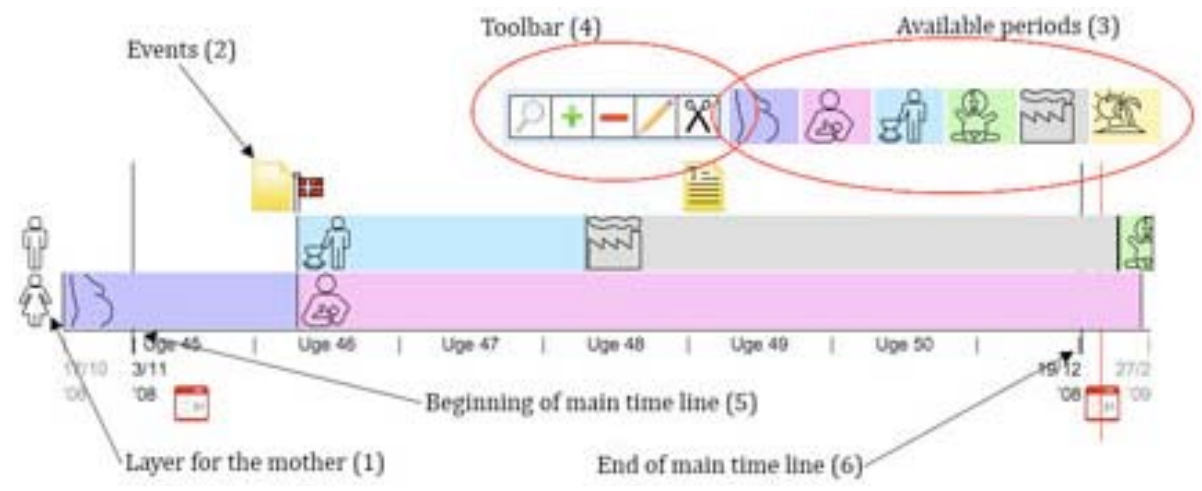

Fig. 1. Screenshot of our prototype

\section{Visualization and Manipulation with Administrative Procedures}

Parental leave is a serious business - jobs are put on hold to care for the newborn, and the family's economy must be taken into consideration. Parental leave is under Danish law supported by the state (and very often additional leave is supported by the employer depending on trade union agreements). The leave is divided into a number of different periods, such as pre- and post-natal, each period governed by different rules. Thus, the combination of leave periods (which excluding pre- and postnatal leave can shared equally between parents) is quite crucial, when a family is planning the time (usually at least a year) after birth. To help parents plan their leave periods in an accessible manner, we have devised a collaborative web-based tool.

During our initial designs, we investigated several approaches to the presentation and overview of administrative procedures. We present in this poster our work on using timelines as an overarching structural mechanism. Maternity leave consists in 
Denmark of different periods of time, where a parent has paid leave of absence from work. Certain parts of this leave can only occur at specific times, and the timeline is well suited to highlight when such periods are applicable. The overall concept is that the timeline should give the applicant a better view of when everything is happening. Our timeline, shown in Figure 1, consists of four layers, the timeline itself, a layer showing periods related to the mother, labeled (1), a layer showing the periods related to the father, and a top layer showing different events, labeled (2), such as birth, expected birth, as well as the documents, which form a critical part of the communication between the parents and the municipality.

The timeline consists of three horizontal sections. The middle section, between (5) and (6), shows a six week time period centered on the current date, and at either end compact sections showing the events which occur outside the main section. One can change the span of the middle section and thereby zoom in and out on the timeline.

The timeline does not only provide visualization and overview of the casework, it is also a tool to interact with and manipulate the information. Interaction with the timeline is done with a combination of direct manipulation and the use of tools selected from a toolbar, labeled (4) in Figure 1.

We have chosen to combine both direct manipulation and tool manipulation because this gives a greater flexibility in the applications supported. Relying only on direct manipulation, where the action executed is chosen by the location or context of the mouse, may restrict the actions available, because only one action can be done at one place, or mouse actions will have to be combined with key presses, giving the user more combinations to remember. We have chosen to include direct manipulation as it gives a nice immediate interaction.

Adding periods of leave is done by dragging periods from the period panel, labeled (3) in Figure 1, and onto the timeline in either the layer for the mother, or the layer for the father. A two-week period will then be added to the respective timeline, starting at the point where the period was dropped. The length of the period can be changed by dragging the ends, making it smaller or larger. Moving the periods is done by clicking a period and moving it to another location. Moving a period off the timeline deletes it. Periods can be cut in two, using the cut tool. This allows the user to move or delete only part of a period.

Events in the timeline, such as birth or application documents, can be either static or allow manipulation. An event such as birth should be static once created and not allow manipulation, whereas events such as application documents should allow manipulation. Events cannot be moved by dragging and the placement on the timeline is decided by internal state of the event.

\section{Prototyping}

We have conducted user evaluations of our design using prototypes throughout our design process. These prototypes have been shown to both citizens and caseworkers from one of the collaborating municipalities. In the early stages of the design process, we employed paper mock-ups to facilitate feedback on our timeline concept, as well as the general interaction and manipulation. A working prototype implementing some of the features of the design has also been developed and evaluated through 
workshops. Both citizens and caseworkers have been given the opportunity to interact with the timeline and associated tools. This process led us to our current prototype which supports dragging periods to the timeline and interacting using tools.

\section{Generalisability and Future Work}

The visualization of casework is not restricted to parental leave and should not only be considered relevant for the outsiders, the citizens, but also for the insiders, the caseworkers. Our timeline design allows for all types of periods and events to be implemented and applied to the timeline. Public work is, at least in the Danish administrative tradition, divided across different departments, such as social, traffic, planning, environment, etc. A case, perceived by the citizen as just one case, may well span several departments and multiple caseworkers. Here, the timeline could be employed to show the current state of the application, letting different periods/layers signify departments or case workers, on whose desk the case (figuratively if not literally) currently resides. This would not only provide the citizens with an insight into the state of their application, but also allow caseworkers to follow cases as they moved to other departments. An example of such a use for the timeline can be found in the ordinary processing of applications (e.g. building permits), which can involve a number of case workers and several departments.

We are currently working on extending the validation of time periods input by the user, so that illegal combinations are caught as early as possible, and also enabling users to see the (financial) consequences of their choices. A simple example of such a needed validation is that you can only qualify for municipal paid leave of absence if you have been employed for 13 weeks in the past year. Some parts of the law apparatus can readily be formalized into a machine decidable system, while other parts will require the experience of a caseworker - we aim with our design to let the caseworkers concentrate on the areas where their expertise is actually needed, rather than being bogged down by trivial, but time consuming tasks that can be handled by our system. We also intend to extend the prototype by implementing more direct manipulation such as allowing dragging the ends of the periods to manipulate length, to allow evaluation of interaction.

\section{References}

1. TimeRime, http://www.timerime.com/

2. TimeLineIndex, http://www. timelineindex.com/

3. TimeGlider, http://www.timeglider.com/

4. SIMILE Timeline Widget, http://www.simile-widgets.org/timeline/ 